EESTI NSV TEADUSTE AKADEEMIA TOIMETISED. XI KOIDE FOUSIKALIS-MATEMAATILISTE JA TEHNILISTE TEADUSTE SEERIA. 1962, NR. 2

ИЗВЕСТИЯ АКАДЕМИИ НАУК ЭСТОНСКОН ССР. ТОМ ХI СЕРИЯ ФИЗИКО-МАТЕМАТИЧЕСКИХ И ТЕХНИЧЕСКИХ НАУК, 1962, N\& 2

\title{
ОБОБЩЕННЫЕ МНОЖИТЕЛИ СХОДИМОСТИ ДЛЯ МЕТОДА ЭЙЛРА-КНОППА
}

\section{Ф. ВИХМАНН}

Последовательность $\left\{s_{k}\right\}$ называется суммируемой методом Эйлера-Кноппа $E^{p}$, если существует предел $\lim \sigma_{v}=\sigma$, где

$v \rightarrow \infty$

$$
\sigma_{v}=\frac{1}{p^{v}} \sum_{k=0}^{v}\left(\begin{array}{l}
v \\
k
\end{array}\right)(p-1)^{v-k_{S}} s_{k} \quad(v=0,1, \ldots) .
$$

Если $p>1$, то метод $E^{p}$ регулярен. Пусть дан еще другой регулярный метод Эйлера-Кноппа $E^{q}$ преобразованием

$$
\tau_{\mathrm{v}}=\frac{1}{q^{v}} \sum_{l=0}^{v}\left(\begin{array}{l}
v \\
l
\end{array}\right)(q-1)^{v-1 t_{l}} \quad(v=0,1, \ldots)
$$

Преобразования (1) и (2) имеют следующие обратные преобразования:

$$
s_{v}=\sum_{k=0}^{v}\left(\begin{array}{l}
v \\
k
\end{array}\right) p^{k}(1-p)^{*-k} \sigma_{k} \quad(v=0,1, \ldots)
$$

и

$$
t_{\mathrm{v}}=\sum_{l=0}^{v}\left(\begin{array}{l}
v \\
l
\end{array}\right) q^{l}(1-q)^{v-l} \tau_{l} \quad(v=0,1, \ldots) .
$$

Определени е. Величины $\varepsilon_{n}(n=0,1, \ldots)$ называются множителями сходимости относительно $\mathfrak{F}^{p}$ u $\mathfrak{F}^{q}$. если ряд $\sum \varepsilon_{n} s_{n} t_{n}$ сходится при каждой $E^{p}$-суммируемой последовательности $\left\{s_{n}\right\}$ и каждой $E^{q}$-суммируемой последовательности $\left\{t_{n}\right\}$. Величины $\varepsilon_{n}(n=0,1, \ldots)$ называются множителями сходимости относительно $E^{p} и E^{q}$, если ряд $\sum \varepsilon_{n} a_{n} b_{n}$ сходится при каждой $E^{p}$-суммируемой последовательности $\left\{\sum_{\nu=0}^{n} a_{v}\right\}$ и $E^{q}$-суммируемой последовательности $\left\{\sum_{\nu=0}^{n} b_{\nu}\right\}$.

В настоящей статье обобщаются некоторые результаты Пейеримхоффа ['], а также Гайера и Пейеримхоффа [], касающиеся простых множителей сходимости для $E^{p}$, на случай обобщенных множителей сходимости. 
Т еорем а 1. Величины $\varepsilon_{n}=(-\Theta)^{n}(n=0,1, \ldots), 0 \leqslant \Theta \leqslant \frac{1}{(2 p-1)(2 q-1)}$, являются множителями сходимости относительно (5 ${ }^{p}$ и ङ $^{q}$.

При доказательстве теоремы используется следующая

Лем м а (Фроли, $\left.\left[{ }^{3}\right]\right)$. Преобразование $z_{n}=\sum_{k, l=0}^{\infty} A_{n k l} x_{k} y_{l} \quad(n=0,1, \ldots)$ nереводит пространство $c_{0} \times c_{0}$ в пространство с тогда $u$ только тогда, когда

$1^{\circ} \lim A_{n k l}=A_{k l} \quad(k, l=0,1, \ldots)$,

$2^{\circ} \sup _{\substack{\|x\| \leqslant 1,\|y\| \leqslant 1,}}\left|\sum_{k, l=0}^{\infty} \mathrm{A}_{n k l} x_{k} y_{l}\right|=O(1)$, zде $x=\left\{x_{k}\right\}, y=\left\{y_{l}\right\}$.
$x, y \in c_{0}$.

Условие $2^{\circ}$ выполнено, если $\sum_{k, l=0}^{\infty}\left|A_{n k l}\right|=O(1)$.

Так как для каждой $E^{p}$-суммируемой последовательности $\left\{s_{v}\right\}$ и $E^{q}$-суммируемой последовательности $\left\{t_{v}\right\}$ известно, что $s_{v}=o\left[(2 p-1)^{v}\right]$ и $t_{v}=o\left[(2 q-1)^{v}\right]$ (см. [ $\left.{ }^{4}\right]$, стр. 228). то достаточно показать, что ряд $\sum \varepsilon_{v} s_{v} t_{v}$ сходится при каждой последовательности $\left\{s_{v}\right\}, E^{p}$-суммируемой к нулю, и каждой последовательности $\left\{t_{v}\right\}, E^{q}$-суммируемой к нулю.

Прннимая во внимание формулы (3) и (4) и обозначая $\max \{k, l\}$ через $(k, l)$, получаем:

$$
\begin{aligned}
& \sum_{\nu=0}^{n} \varepsilon_{\nu} s_{\nu} t_{\nu}=\sum_{\nu=0}^{n}(-\Theta)^{\nu} \sum_{k, l=0}^{\nu}\left(\begin{array}{l}
\nu \\
k
\end{array}\right)\left(\begin{array}{l}
\nu \\
l
\end{array}\right) p^{k} q^{l}(1-p)^{\nu-k}(1-q)^{\nu-l} \sigma_{k} \tau_{l}= \\
& =\sum_{k, l=0}^{n} p^{k} q^{l} \sigma_{k} \tau_{l} \sum_{\nu=0}^{n-(k, l)}(-1)^{v+(k, l)}\left({ }^{v}+\underset{k}{(k, l)}\right)\left({ }^{v}+(k, l)\right)(1-p)^{v+(k, l)-k}(1-q)^{\nu}+(k, l)-l \Theta^{v+(k, l)}= \\
& =\sum_{k, l=0}^{n} \sigma_{k} \tau_{l} p^{k} q^{l}(1-p)^{(k, l)-k}(1-q)^{(k, l)-l} \Theta^{(k, l)}\left\{\sum_{\nu=0}^{\infty}-\sum_{\nu=n-(k, l)+1}^{\infty}\right\}(-1)^{\nu+(k, l)}\left({ }^{\nu}+(k, l)\right) . \\
& \cdot\left({ }^{v}+(k, l)\right)[(1-p)(1-q) \Theta]^{v}=\sum_{k, l=0}^{n} A_{k l} \sigma_{k} \tau_{l}-\sum_{k, l=0}^{n} B_{n k l} \sigma_{k} \tau_{l} .
\end{aligned}
$$

Покажем, что существуют пределы при $n \rightarrow \infty$ обеих сумм при каждой последовательности $\left\{\sigma_{k}\right\} \in c_{0}$ и каждой последовательности $\left\{\tau_{l}\right\} \in c_{0}$. Для этого величины $A_{n k 1}\left[A_{t k l}=A_{k l}(k, l \leqslant n), \quad A_{n k l}=0\right.$ в остальных случаях $]$ и $B_{n k l}(k, l, n=0,1, \ldots)$ должны удовлетворять условиям леммы.

Обозначаем

$$
y=(1-p)(1-q) \Theta
$$

Ччевидно, $0 \leqslant y \leqslant 1$. 
1. Покажем, что условия леммы выполнены для величин $A_{n k l}(n, k, l)$. Пусть $k \geqslant l$. Обозначаем сумму ряда $\sum_{\nu=0}^{\infty}(-1)^{\nu}{ }^{+k}\left({ }^{\nu}+k\right)\left({ }^{\nu}+k\right) y^{\nu}$ через $P_{k l}(y)$.

Тогда

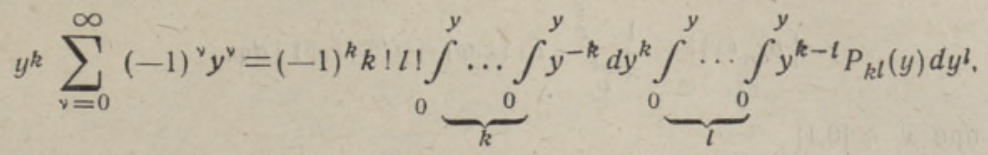

откуда

$P_{k l}(y)=\frac{(-1)^{k} y^{l-k}}{l !} \frac{d^{l}}{d y^{l}}\left[\frac{y^{k}}{(1+y)^{k+1}}\right]=\frac{(-1)^{k}}{(1+y)^{k+1}} \sum_{\nu=0}^{l}(-1)^{\nu}\left(\begin{array}{l}l \\ \nu\end{array}\right)\left(\begin{array}{c}k+\nu \\ l\end{array}\right)\left(\frac{y}{1+y}\right)^{\nu}$

Обозначаем $\frac{y}{1+y}$ через $x$. Очевидно, $0 \leqslant x<0,5$.

Определяем полиномы $p_{l n}(x)$ для $l=0,1, \ldots$ :

$$
\begin{array}{r}
p_{l n}(x)=\frac{(-1)^{n}}{\left(\begin{array}{c}
n+l \\
t
\end{array}\right)} \sum_{\nu=0}^{n}(-1)^{\nu}\left(\begin{array}{l}
n \\
\nu
\end{array}\right)\left(\begin{array}{l}
n+l+\nu \\
n
\end{array}\right) x^{\nu}= \\
=\frac{(-1)^{n} x^{-l}}{\left(\begin{array}{c}
n+l \\
n
\end{array}\right) n !} \frac{d^{n}}{d x^{n}}\left[x^{n+l}(1-x)^{n}\right] \quad(n=0,1, \ldots) .
\end{array}
$$

Легкंо убеднться, что полиномы $p_{l n}(x)(n=0,1, \ldots)$ при фиксированном $l$ ортогональны относительно веса $x^{l}$ на интервале $(0,1)$.

Нетрудно увидеть, что имеет место интегральное представление

$$
p_{\text {on }}(x)=\frac{(-1)^{n}}{2 \pi i} \int_{C} \frac{z^{n}(z-1)^{n}}{(z-x)^{n+1}} d z \quad(n=0,1, \ldots) \text {, }
$$

где $C$ - замкнутый контур, окружающий точку $x$. Пусть, в частности, $x$ - действительное число, $|x|>1$, а $C-$ окружность с центром в точке $x$ и радиусом $\sqrt{x(x-1)}$. Полагая $\quad z=x+\sqrt{x(x-1)} e^{i \varphi}, \quad$ находим $\quad z(z-1)=e^{i \varphi} \sqrt{x(x-1)}[(2 x-1)+$ $+2 \sqrt{x(x-1)} \cos \varphi]$ и, следовательно,

$$
p_{\text {on }}(x)=\frac{(-1)^{n}}{\pi} \int_{0}^{\pi}[(2 x-1)+2 \sqrt{x(x-1)} \cos \varphi]^{n} d \varphi \text {. }
$$

Так как здесь слева и справа стоят полиномы от $x$, то последнее равенство, полученное нами для $|x|>1$, верно для всех действительных $x$.

Покажем, что для $x \in[0,1]$ имеем $\left|p_{\text {on }}(x)\right| \leqslant 1 \quad(n=0,1, \ldots)$. Так как ·

$$
\int_{0}^{\pi} \cos ^{2 n+1} x d x=0 \quad(n=0,1, \ldots)
$$

To

$$
p_{o n}(x)=\frac{(-1)^{n}}{\pi} \int_{0}^{\pi}[(2 x-1)+2 i \sqrt{x(1-x)} \cos \varphi]^{n} d \varphi=\frac{(-1)^{n}}{\pi} j_{-n}^{\pi}\left[F_{1}(x . D)+i F_{2}(x .)\right] d \varphi \text {. }
$$


где

$$
\int_{0}^{\pi} F_{1}(x, \varphi) d \varphi=0, \quad p_{o n}(x)=\frac{(-1)^{n}}{\pi} \int_{0}^{\pi} F_{2}(x, \varphi) d \varphi .
$$

Следовательно,

$$
\left|p_{o n}(x)\right| \leqslant \frac{1}{\pi} \int_{0}^{\pi}\left|F_{1}(x, \varphi)+i F_{2}(x, \varphi)\right| d \varphi .
$$

Поскольку при $x \in[0,1]$

$$
|(2 x-1)+i \sqrt{x(1-x)} \cos \varphi|=\left[(2 x-1)^{2} \sin ^{2} \varphi+\cos ^{2} \varphi\right]^{\frac{1}{2}} \leqslant 1,
$$

Tо

$$
-\left|p_{u n}(x)\right| \leqslant 1 \quad(x \in[0,1]) .
$$

Покажем теперь, что $\left|p_{l n}(x)\right| \leqslant 1 \quad(l, n=0,1, \ldots)$ для $x \in[0,1]$. Для этого докажем справедливость формулы

$$
\begin{array}{r}
p_{l n}(x)=\frac{1}{\left(\begin{array}{l}
l+n \\
l
\end{array}\right)^{2}} \sum_{\nu=0}^{n}(-1)^{v}\left[\left({ }^{l+n-v}\right)^{2}-\left({ }_{l}^{l+n-\nu-1}\right)^{2}\right] p_{l-1, n-\nu}(x) . \\
(l=1,2, \ldots ; n=0,1, \ldots) .
\end{array}
$$

Формула (8) верна, очевидно, тогда и только тогда, когда коэффициенты при $x^{v}(v=0,1, \ldots, n)$ слева и справа в равенстве (8) равны. Вставим вместо $p_{l n}(x)$ и $p_{l-1, i}(x) \quad(i=0,1, \ldots, n)$ их выражения из (6). Если $v=0$, то из (8) получаем

$$
(-1)^{n}=\frac{(-1)^{n}}{\left({ }^{l+n}\right)^{2}} \sum_{\nu=0}^{n}\left[\left({ }^{l+n-v}\right)^{2}-\left({ }^{l+n-v-1}\right)^{2}\right]
$$

справедливость которого очевидна. Если $v \neq 0$, то при каждом $n \geqslant v$ равенство коэффициентов при $x^{2}$ выражается соотношением

$$
\frac{(-1)^{n+v}\left(\begin{array}{l}
n \\
v
\end{array}\right)\left(\begin{array}{l}
n+l+v \\
n
\end{array}\right)}{\left(\begin{array}{c}
n+l \\
n
\end{array}\right)}=\frac{1}{\left(\begin{array}{c}
n+l \\
n
\end{array}\right)^{2}} \sum_{i=v}^{n}(-1)^{n+v} \frac{\left(\begin{array}{l}
i \\
v
\end{array}\right)\left(\begin{array}{l}
i+v+l-1 \\
i
\end{array}\right)}{\left(\begin{array}{c}
i+l-1 \\
i
\end{array}\right)}\left[\left(\begin{array}{l}
l+i \\
l
\end{array}\right)^{2}-\left(\begin{array}{l}
l+i-1 \\
l
\end{array}\right)^{2}\right],
$$

или

$$
\frac{(n+l) !(n+l+v) !}{n !(n-v) !(l+v)}=\sum_{i=v}^{n} \frac{(l+i-1) !(l+v+i-1) !(l+2 i)}{i !(i-v) !}
$$

Очевидно, что последнее равенство справедливо при $n=v$. Предположим его справедлмвость при всех $n, v \leqslant n \leqslant m$, и покажем, что оно останется в силе при $n=m+1$.

Действительно,

$$
\begin{gathered}
\sum_{i=v}^{m+1} \frac{(l+i-1) !(l+v+i-1) !(l+2 i)}{i !(i-v) !}=\frac{(m+l) !(m+l+v) !}{m !(m-v) !(l+v)}+ \\
+\frac{(m+l) !(m+l+v) ![2(m+1)+l]}{(m+1) !(m+1-v) !}=\frac{(m+l) !(m+l+v) !}{(m+1) !(m+1-v) !(l+v)} . \\
\cdot\{(m+1)(m+1-v)+(l+v)[2(m+1)+l]\}=\frac{(m+1+l) !(m+1+l+v) !}{(m+1) !(m+1-v) !(l+v)} .
\end{gathered}
$$


Так как $\frac{1}{\left(\begin{array}{l}l+n \\ l\end{array}\right)^{2}} \sum_{\nu=0}^{n}\left[\left({ }^{l+n-v}\right)^{2}-\left(\begin{array}{c}l+n-v-1 \\ l\end{array}\right)^{2}\right]=1$, то, ввнду (7) нз формуль (8) вытекает $\left|p_{\text {Ln }}(x)\right| \leqslant 1(l, n=0,1, \ldots)$ для $x \in[0,1]$.

Теперь уже легко показать, что условие $2^{\circ}$ леммы выполнено для величин $A_{n k l}(k, t, n=0,1, \ldots)$. Так как

$\sum_{k=0}^{n} \sum_{l=0}^{k}\left|A_{k l}\right|=\sum_{k=0}^{n} \frac{p^{k} \Theta^{k}}{(1+y)^{k+1}} \sum_{l=0}^{k}\left(\begin{array}{l}k \\ l\end{array}\right) q^{l}(q-1)^{k-l}\left|\frac{1}{\left(\begin{array}{l}k \\ l\end{array}\right)} \sum_{\nu=0}^{l}(-1)^{\nu}\left(\begin{array}{l}l \\ v\end{array}\right)\left(\begin{array}{c}k+\nu \\ l\end{array}\right)\left(\frac{y}{1+y}\right)^{\nu}\right|=$ $=\sum_{k=0}^{n} \frac{p^{k} \Theta^{k}}{(1+y)^{k+1}} \sum_{l=0}^{k}\left(\begin{array}{l}k \\ l\end{array}\right) q^{l}(q-1)^{k-l}\left|p_{k-l, l}(x)\right|=O(1) \sum_{k=0}^{n} \frac{p^{k}(2 q-1)^{k} \Theta^{k}}{(1+y)^{k+1}}=O(1)$ и аналогично $\sum_{l=0}^{n} \sum_{k=0}^{l}\left|A_{k l}\right|=O(1)$, то $\sum_{k, l=0}^{n}\left|A_{k l}\right|=O(1)$. Условие $1^{\circ}$ леммы автоматически выполнено.

2. Покажем, что для величнн $B_{n k l}(k, l, n=0,1, \ldots)$ выполнены условия леммы. Пусть $k \geqslant l$. Обозначаем через $P_{n k l}(y)$ сумму ряда $\sum_{v=n=k}^{\infty}(-1)^{\nu+k}\left({ }^{\nu}+k\right)\left({ }^{\nu}+k\right) y^{\nu}$, где $y$ определено формулой (5). Рассматривая $y$ как переменную, можно выписать равенство

$$
\frac{(-1)^{k}}{l !} \sum_{\nu=n=k+1}^{\infty}(-1)^{\nu}\left(\nu_{k}^{\nu+k}\right) y^{\nu+k}=\int_{0}^{y} \ldots \int_{l}^{y} y^{k-l P_{n k l}}(y) d y l^{l}
$$

Представим здесь остаточный член ряда $\sum_{\nu=0}^{\infty}(-1)^{\nu}\left({ }^{v}+k\right) y^{\nu}$ в форме Лагранжа:

$$
\sum_{\nu=n+1}^{\infty}(-1)^{\nu}\left({ }_{k}^{\nu+k}\right) y^{\nu}=\frac{y^{n-k+1}}{(n-k+1) !}\left[\frac{d^{n-k+1}}{d \eta^{n-k+1}}\left(\frac{1}{1+\eta}\right)^{k+1}\right]_{\eta=\delta y} \text {, }
$$

где $0<\delta<1$. Следовательно,

$$
\begin{aligned}
P_{n k l}(y) & =\frac{(-1)^{k}\left({ }^{n+1}\right)}{(n-k+1) !} y^{n-k+1}\left[\frac{d^{n-k+1}}{d \eta^{n-k+1}}\left(\frac{1}{1+\eta}\right)^{k+1}\right]_{\eta=\delta y}= \\
& =\left.(-1)^{n+1}\left({ }^{n+1}{ }_{k}\right)\left({ }^{n+1} l^{-1}\right) y^{n-k+1}(1+\eta)^{-n-2}\right|_{\eta=\delta y},
\end{aligned}
$$

откуда

$$
\left|P_{n k l}(y)\right|=\odot(1)\left(\begin{array}{c}
n+1 \\
k
\end{array}\right)\left(\begin{array}{c}
n+1 \\
l
\end{array}\right) y^{n-k+1} \quad(0 \leqslant y<1) .
$$

Аналогично, если $l \geqslant k$, то

$$
\left|P_{n k l}(y)\right|=O(1)\left(\begin{array}{c}
n+1 \\
k
\end{array}\right)\left({ }^{n+1} l^{-1}\right) y^{n-l+1} \quad(0 \leqslant y<1) .
$$




$$
\begin{aligned}
& \text { Оценим выражение } \sum_{k, l=0}^{n}\left|B_{n k l}\right|: \\
& \sum_{k, l=0}^{n}\left|B_{n k l}\right|=O(1)\left\{\sum_{k=0}^{n}\left({ }^{n+1}\right) \frac{p^{k} y^{n-k+1}}{(2 p-1)^{k}(2 q-1)^{k}} \sum_{l=0}^{k-1}\left({ }^{n+1}\right) q^{l}(q-1)^{k-l}+\right. \\
& \left.+\sum_{l=0}^{n}\left({ }^{n+1}{ }^{l}\right) \frac{q^{l} y^{n-l+1}}{(2 p-1)^{l}(2 q-1)^{l}} \sum_{k=0}^{l}\left({ }^{n+1}{ }_{k}\right) p^{k}(p-1)^{l-k}\right\}= \\
& =\frac{O(1)}{(2 p-1)^{n+1}(2 q-1)^{n+1}} \sum_{k, l=0}^{n}\left({ }^{n+1}\right)\left(^{n}{ }_{l}+1\right) p^{k} q^{l}(p-1)^{n+1-k}(q-1)^{n+1-l}= \\
& =\frac{O(1)}{(2 p-1)^{n+1}(2 q-1)^{n+1}\left[(2 p-1)^{n+1}-p^{n+1}\right]\left[(2 q-1)^{n+1}-q^{n+1}\right]=O(1) .}
\end{aligned}
$$

Значит, условие $2^{\circ}$ леммы выполнено, а выполнение условия $1^{\circ}$ тривиально.

Те орема 2. Величины $\varepsilon_{n}=(-\Theta)^{n}(n=0,1, \ldots), 0 \leqslant \Theta \leqslant \frac{1}{(2 p-1)(2 q-1)}$, являются множителями сходимости относительно $E^{p}$ и $E^{q}$.

Д ок а з тельство Справедлива формула

$$
\sum_{v=0}^{n} \varepsilon_{\mathrm{v}} a_{\mathrm{v}} b_{\mathrm{v}}=\sum_{\mathrm{v}=0}^{n} \varepsilon_{\mathrm{v}} s_{\mathrm{v}} t_{\mathrm{v}}+\sum_{\mathrm{v}=1}^{n} \varepsilon_{\mathrm{v}} s_{\mathrm{v}-1} t_{\mathrm{v}-1}-\sum_{\nu=1}^{n} \varepsilon_{\mathrm{v}} s_{\mathrm{v}} t_{\mathrm{v}-1}-\sum_{\nu=1}^{n} \varepsilon_{\mathrm{v}} S_{\mathrm{v}-1} t_{\mathrm{v}},
$$

где $s_{\nu}=\sum_{\mu=0}^{\nu} a_{\mu}$ и $t_{\nu}=\sum_{\mu=0}^{\nu} b_{\mu}$.

Так как регулярные методы Эйлера-Кноппа $E^{p}$ и $E^{q}$ транслятивны, то при каждой $E^{p}$-суммируемой последователюности $\left\{s_{0}, s_{1}, \ldots\right\}$ также $E^{p}$-суммируема и последовательность $\left\{0, s_{0}, s_{1}, \ldots\right\}$, а при каждой $E^{q}$-суммируемой последовательности $\left\{t_{0}, t_{1}, \ldots\right\}$ также $E^{7}$-суммируема и последовательность $\left\{0, t_{0}, t_{1}, \ldots\right\}$. Значит, по теореме 1 , справа в равенстве (9) все пределы при $n \rightarrow \infty$ существуют при каждой $E^{p}$-суммируемой последовательности $\left\{s_{v}\right\}$ и каждой $E^{q}$-суммируемой последовательности $\left\{t_{v}\right\}$, что и доказывает теорему.

При мечание. Величины $\varepsilon_{n}=\frac{1}{(2 p-1)^{n}(2 q-1)^{n}}(n=0,1, \ldots)$ не являются множителями сходимости относительно $\mathfrak{5}^{p}$ и $\mathfrak{F}^{q}$. Действительно, иначе ряд $\sum \varepsilon_{v} s_{v} t_{v}$ сходился бы при каждой $E^{p}$-суммируемой последовательности $\left\{s_{v}\right\}$ и каждой $E^{q}$-суммируемой последовательности $\left\{t_{\nu}\right\}$. Но тогда должно выполняться условие $2^{\circ}$ леммы

$$
\begin{aligned}
& A_{n}=\sup \left|\sum_{\nu=0}^{n} \varepsilon_{v} \sum_{k, l=0}^{\nu}\left(\begin{array}{l}
v \\
k
\end{array}\right)\left(\begin{array}{l}
\nu \\
l
\end{array}\right) p^{k} q^{l}(1-p)^{2-k}(1-q)^{\nu-l} \sigma_{k} \tau_{l}\right|=O(1) \\
& \|\sigma\| \leqslant 1, \\
& \quad\|\tau\| \leqslant 1, \\
& \quad \sigma, \tau \in c_{0} .
\end{aligned}
$$


Возьмем злесь $\sigma=\left\{\sigma_{k}\right\}=\left\{1,-1,1, \ldots,(-1)^{n}, 0,0, \ldots\right\}$ и $\tau=\left\{\tau_{l}\right\}=$ $=\left\{1,-1,1, \ldots,(-1)^{n}, 0,0, \ldots\right\}$. Тогда $A_{n} \geqslant n+1$, что не совместимо с условием $A_{n}=O(1)$.

\section{Л И Т Р Р А У Р А}

1. A. Peyerimhofi, Konvergenzfaktoren beim Euler-Knoppschen Limitierungsverfahren, Math. Z., 55, 288-291.

2. D. Ga ier, A. P e y er imhoff, Summierbarkeitsfaktoren bei Eulerschen Reihentransformationen, Math. Z., 58, 232-242.

3. P. A. Frale igh, Regular bilinear transformations of sequences, Amer. Journ. Math., 53 (1931), 667-709.

4. Г. Х а рди, Расходящиеся ряды, М. (1951)

Тартуский государственный университет

Поступила в редакцию

24. IV 1961

\section{OLDISTATUD SUMMEERUVUSTEGURID EULERI-KNOPPI MENETLUSE KORRAL}

\section{F. Vichmann}

\section{Resümee}

Olgu $E^{p}$ ja $E^{q}$ Euleri-Knoppi summeerimismenetlused. Reaalarve $\varepsilon_{n}(n=0,1, \ldots)$ nime-

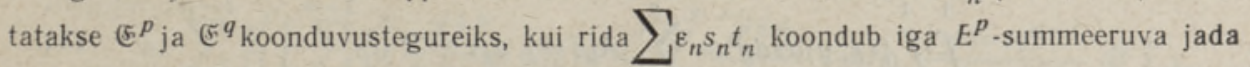
$\left\{\mathrm{s}_{n}\right\}$ ja iga $E^{q}$-summeeruva jada $\left\{t_{n}\right\}$ korral.

Artiklis tõestatakse, et arvud $\varepsilon_{n}=(-\Theta)^{n}(n=0,1, \ldots), 0 \leqslant \Theta \leqslant \frac{1}{(2 p-1)(2 q-1)}$, on $\mathscr{E}^{p}$ ja $\xi^{q}$ koonduvustegurid $(p, q>1)$.

Tartu Riiklik Olikool

Saabus toimetusse 24. IV 1961

\section{VERALLGEMEINERTE KONVERGENZFAKTOREN BEIM EULER-KNOPPSCHEN LIMITIERUNGSVERFAHREN}

\section{F. Vichmann}

\section{Zusammenfassung}

$E^{p}$ und $E^{q}$ seien Euler-Knoppsche Limitierungsverfahren. Reelle Zahlen $\varepsilon_{n}(n=0,1, \ldots)$ nennt man Konvergenzfaktoren der $\mathfrak{}^{p}$. und $\mathfrak{\digamma}^{q}$, wenn für alle $E^{p}$-limitierbaren Folgen $\left\{s_{n}\right\}$ und für alle $E^{q}$-limitierbaren Folgen $\left\{t_{n}\right.$ : stets die Reihe $\sum \varepsilon_{n} s_{n} t_{n}$ konvergiert.

Im Aufsatz wird bewiesen, dass die Zahlen $\varepsilon_{n}=(-\Theta)^{n}(n=0,1, \ldots)$, $0 \leqslant \theta \leqslant \frac{1}{(2 p-1)(2 q-1)}$ Konvergenzfaktoren der ( $^{p}$ und (F $^{q}$ sind $(p, q>1)$. 